\title{
Gasto para el desarrollo rural en México y Presupuesto Base Cero, 2016 ${ }^{1}$ Expenditure for Rural Development in Mexico and Zero Base Budget, 2016
}

\section{Journal of Economic Literature (JEL):}

E63, H61, O23

Palabras clave:

Gasto

Crecimiento

Pobreza

Desarrollo

Keywords:

Spending

Growth

Poverty

Develoment

\section{Resumen}

Este artículo analiza la evolución de los métodos presupuestarios en México. Se asume que el presupuesto es una de las herramientas más importantes del desarrollo, puesto que concentra un conjunto de programas productivos y sociales para atender los problemas nacionales. Con una breve revisión de las técnicas presupuestarias seguidas en México entre 1935 y 2015, se llega hasta el actual Presu-

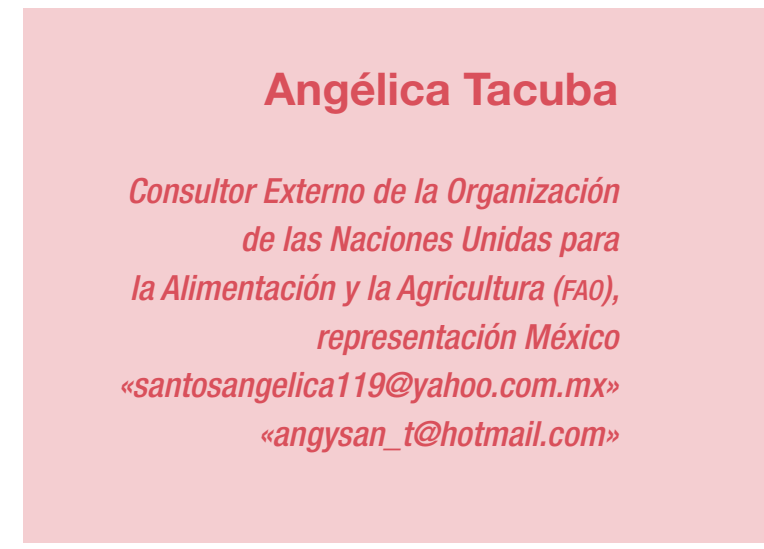
puesto Basado en Resultados (PBR), producto de la búsqueda de una mayor eficiencia en el diseño y ejercicio del gasto público. El análisis destaca que junto a esta técnica subyace la forma inercial de gastar (incrementalista) que nunca cuestiona los resultados de los programas, y que tampoco tiene un procedimiento para evaluar los impactos de los mismos. Ese escenario determina el modelo de gasto seguido en materia rural, su eficiencia y sus resultados de desarrollo; la reciente propuesta del Gobierno Federal de implementar el Presupuesto Base Cero (PBC) para el ejercicio fiscal 2016, abre la posibilidad de explorar las implicaciones del nuevo método para el diseño y ejercicio del gasto destinado al desarrollo rural. Ello, en razón de la desigualdad social, la pobreza y el estancamiento económico prevaleciente en ese territorio que se muestran como los justificantes más importantes para la reorientación del modo de gastar.

1 Este artículo es resultado de la tesis de doctorado: Gasto público y desarrollo rural en México, 1990-2013, realizada en el Posgrado de Economía UNAM. El tema obtuvo mención honorifica y se publicará bajo la plataforma Ciencia Nueva Doctorados de la UNAM. Se agradece a todos los integrantes del comité de tutores, en especial, a Carlos Tello Macías, quien fungió como tutor principal y aportó mucho en la revisión del tema aquí abordado. 


\section{Abstract}

This article analyzes the evolution of budgetary methods in Mexico. It is assumed that the budget is one of the most important tools of development, since it concentrates a set of productive and social programs to address national problems. With a brief review of budgeting techniques followed in Mexico between 1935 and 2015, you reach the current results-based budgeting (RBB), a product of the search for greater efficiency in the design of public expenditure. The analysis shows that this technique lies with how to spend inertial (incrementalism) that never questions the results of the programs, nor has a procedure for assessing the impacts thereof. That scenario determines the expense model followed in rural area, their efficiency and development results; the recent proposal by the Federal Government to implement the Zero Base Budget (ZBB) for fiscal year 2016, opens the possibility of exploring the implications of the new method for the design and execution of spending for rural development. This, because of social inequality, poverty and economic stagnation prevailing in that territory shown as the most important justifications for redirecting spending mode.

Introducción con el gasto. Presupuestar significa planear estratégicamente, definir los objetivos sociales, el tipo de políticas a ejercer, y el uso y disposición de los recursos. Involucra aspectos de control administrativo (los fondos son usados efectivamente en el logro de metas), y de control operacional (las tareas específicas de cada departamento son llevadas a cabo eficientemente). ${ }^{2}$

La definición del presupuesto nace de las finanzas públicas como ciencia que trata de la obtención de los ingresos, su inversión, y los efectos esperados en la actividad económica y social de los países. ${ }^{3}$

La influencia del presupuesto público sobre el desarrollo, visto como el proceso de crecimiento sostenido combinado con justicia social, ha sido analizada desde distintas vertientes. Así, por ejemplo, encontramos en Adam Smith, una agenda del Estado donde la inversión en obras para facilitar el comercio y la educación es un instrumento para aumentar la riqueza de las naciones. ${ }^{4}$ En Keynes, encontramos el concepto del multiplicador del gasto, que se refiere a los impactos de un aumento de la inversión pública sobre el empleo, el ingreso y el consumo. ${ }^{5}$ En Hirschman, halla-

2 Freemont Lyden y Miller, Ernest, Presupuesto público. Planeación, evaluación y control de programas, México, Trillas, 1983.

3 Ibid, p. 73.

4 Adam Smith, La riqueza de las naciones. Madrid, España, Alianza Editorial, 2002.

5 John Keynes, Teoría general de la ocupación, el interés y el dinero, México, Fondo de Cultura Económica, 2000. Vease tambien, Federico Novelo, Invitación a Keynes, México, Universidad Autonóma Metropolitana, Fondo de Cultura Económica, 1997. 
mos los eslabonamientos fiscales, como las secuencias de inversión que siguen a un incremento del gasto público. 6

En el medio rural, donde el Producto Interno Bruto (PIB) agropecuario crece a una tasa de $1.9 \%$ anual, y donde habitan 25 millones de personas, de las

En el medio rural las funciones de promoción del crecimiento y redistribución del ingreso del presupuesto son mucho más importantes cuales, 10.9 millones se encuentran en pobreza moderada, y 5.8 millones en pobreza extrema ( $66.8 \%$ del total), las funciones de promoción del crecimiento y redistribución del ingreso del presupuesto son mucho más importantes. Se exige una mayor correspondencia entre cantidades de gasto y calidad de los programas. ${ }^{7}$

La técnica presupuestaria seguida a nivel federal, afecta el diseño y ejecución del presupuesto rural y los resultados esperados de la inversión pública. ${ }^{8}$ A partir del surgimiento de la primera ley presupuestaria en 1935 , la manera de presupuestar en México se ha modificado cuando menos cuatro veces, impactando el ejercicio del gasto público rural.

\section{Métodos presupuestarios en México, 1935-2015}

Entre 1935 y 2015 se han puesto en práctica, tres métodos presupuestarios en México. A partir del 2016, se inicia uno nuevo.

Presupuesto por objeto de gasto. Esta técnica se aplicó entre 1935 (año en que se promulgó la Ley Orgánica del Presupuesto de la Federación), y 1976. El presupuesto con base en objeto del gasto funcionó a partir de metas de crecimiento en una etapa en la que el desarrollo nacional y la industrialización se fijaron como prioridad. De ese modo, las cantidades de inversión pública fueron crecientes. La técnica consistía en registrar de manera ordenada y sistemática, cada capítulo, concepto y partida de gasto con base en objetivos nacionales de desarrollo.

Presupuesto por programas. Empezó a instrumentarse en 1977 una vez que entró en vigor la nueva ley del presupuesto que sustituyó a la de 1935. Perduró hasta la década de los noventa y se diferenció la técnica por objeto del gasto, en que comenzó a darle importancia a las cantidades de inversión (y contratación de deuda) para lograr los objetivos de política pública, y a los procesos de definición e inclusión de

6 Para Hirschman un eslabonamiento es una secuencia característica, más o menos imperativa de decisiones de inversión que ocurren en el curso de la industrialización y del desarrollo de un país, y que puede aplicarse los efectos de la inversión pública. Albert Hirschman, Enfoques alternativos sobre la sociedad de mercado y otros enfoques recientes, México, Fondo de Cultura Económica, 1989.

7 Para Richard Musgrave, uno de los clásicos de las finanzas públicas, los tres deberes que tiene el Estado en cualquier economía, abarcan: 1) la promoción del crecimiento económico, mediante la inversión en obras públicas y los sectores productivos; 2) redistribución del ingreso, a través del gasto social incluyendo subsidios e impuestos; 3) estabilización macroeconómica, utilizando el gasto con fines contracíclicos en fases de recesión. Richard Musgrave, Principios de determinación del presupuesto. En Francisco Dominguez, Política fiscal en acción, Madrid, Instituto de Estudios Fiscales, 1973.

8 El presupuesto público abarca gasto e ingreso del gobierno. En el presente trabajo sólo se estudia lo relativo al gasto y se utiliza como sinónimo de presupuesto a lo largo del texto. 
programas. Se dio más énfasis al contraste costo-beneficio por partida de gasto, mecanismo que se profundizó después de la crisis de 1982, y que condujo al ajuste fiscal y a la privatización de empresas públicas.

Presupuesto con base en resultados (PBR). La exploración de este método inició a finales de los noventa y se empezó a aplicar en 2006 con la entrada en vigor de Ley Federal de Presupuesto y Responsabilidad Hacendaria (LFPRH) de ese año. El PBR resultó de la definición de un Sistema de Evaluación del Desempeño (SED) y de Gestión por Resultados (GPR) propuesto por los países de la Organización para la Cooperación y el Desarrollo Económico (OCDE), cuyos objetivos eran mejorar la gestión de los recursos públicos y proveer mejores bienes a la población.

El punto más destacable del PBR fue, que, teniendo el objetivo de iniciar con la estimación de los impactos del gasto, introdujo la evaluación de programas en función a indicadores estratégicos y de gestión, vinculados con metas y objetivos entre dependencias y Planes Nacionales de Desarrollo (PND). ${ }^{9}$ La introducción de las evaluaciones fortaleció el principio general de la calidad del gasto como medida para cubrir eficazmente sus fines colectivos y se adaptó bien al criterio de estabilidad macroeconómica iniciado en la década de 1980, en el que la contracción del gasto debería acompañarse de una mayor eficiencia en el diseño y ejecución de programas.

La normatividad derivada de La GPR y del SED obligó a todos los programas sujetos a reglas de operación, incluidos los de naturaleza rural, a someterse a evaluaciones periódicas. ${ }^{10}$ Para ello, se generó la Metodología de Marco Lógico (MML) como un proceso de determinación de objetivos de los programas, propósitos, metas e indicadores para la medición de sus resultados, los cuales, se concentraron en una Matriz de Indicadores de Resultados (MIR) por programa. ${ }^{11}$

El impacto del PBR sobre el presupuesto rural fue la construcción de la MIR para los programas con reglas de operación o lineamientos, en particular aquellos inmersos en objetivos nacionales prioritarios, por ejemplo, el caso del Programa de Desarrollo Humano Oportunidades, en el combate a la pobreza y del Programa de Apoyos Directos al campo, en la producción de granos básicos. Más recientemente, con el inicio del sexenio 2012-2018, los 66 programas incluidos en la Cruzada Nacional contra el Hambre (CNCH).

9 Para una mayor comprensión sobre los criterios del SED y la metodología para la construcción de indicadores estratégicos y de gestión, véase: Secretaría de Hacienda y Crédito Público, Guía para la construcción de indicadores estratégicos, México, SHCP, 2011.

10 Las reglas de operación, son las disposiciones que especifican la forma de operar de un programa. Proporcionan la información sobre quiénes son los sujetos de recibir el apoyo (población objetivo), los requisitos, los objetivos del programa, el procedimiento de solicitud y operación, así como obligaciones de los beneficiarios, los derechos y las sanciones, lbid.

11 La Metodología de Marco Lógico (MML) es una herramienta analítica que concentra en una matriz el objetivo general, los objetivos específicos, los resultados esperados, las actividades para lograr esos resultados, los recursos necesarios y los indicadores para medir lo esperado. Tiene sus antecedentes en la década de los sesenta cuando se introdujo para planificar los proyectos de desarrollo orientados a resultados. Ibid. 
De los 800 programas a nivel federal, 267 cuentan con reglas de operación; de los 130 a escala rural, aproximadamente 30 cuentan con reglas de operación. ${ }^{12}$ Las evaluaciones en estos casos, han sido muy útiles para mejorar la parte del Las evaluaciones diseño de los programas (objetivos, metas, población potencial, población han sido muy útiles objetivo y focalización); la operación (montos y tipos de apoyo, ejecutores, para mejorar la temporalidad, entre otros); y los impactos esperados (definición de indicaparte del diseño de los programas, la operación y los dores medibles que faciliten la estimación de resultados). ${ }^{13}$

Con todos sus avances y ventajas, el PBR es una técnica presupuestal aplicada parcialmente en México. Una primera explicación tiene que ver impactos esperados

con las dificultades operativas para evaluar la totalidad de los programas públicos y sus componentes. ${ }^{14}$

Una segunda, se relaciona con las inercias y normas informales que sobreviven a la mayoría de las reformas presupuestarias. El objetivo principal del PBR fue el de transformar la forma de presupuestar lo que no es sencillo y de corto plazo. Cualquier cambio conlleva la redefinición de instrumentos técnicos y de valores; pero es probable que los valores inicialmente formulados sean desviados hacia fines no colectivos. 15

Una tercera explicación, tiene que ver con la naturaleza política del presupuesto. El presupuesto es un proceso político porque en su preparación y operación, participan distintos actores sociales que tienen visiones divergentes sobre cómo estructurar políticas y programas para resolver los problemas sociales. Esto rara vez se cumple como un ejercicio sensato y está generalmente asociado a la obtención de rentas partidistas y clientelares, lo que vicia todo el trayecto de preparación y ejecución del presupuesto.

Debe reconocerse que en medio de un contexto de intereses e inercias, la aplicación del PBR en México ha tenido sus respectivas retribuciones en la mejora de los programas evaluados, entre ellos, varios dirigidos a la población rural; también, en la construcción de sus respectivas MIR donde de manera esquemática se trazan metas, objetivos e indicadores de medición de resultados.

12 Secretaría de Hacienda y Crédito Público, Manual de Presupuesto 2015, Catalogo de Programas Sujetos a Reglas de Operación, México, 2015.

13 El PBR trajo consigo la implementación de varios tipos de evaluación. Las específicas de desempeño muestran el avance en el cumplimiento de los objetivos y metas programadas de los programas sociales; las Complementarias son de aplicación opcional de acuerdo con las necesidades e intereses de las dependencias y entidades, con el fin de mejorar su gestión y obtener evidencia adicional sobre su desempeño; las de Consistencia y Resultados hacen un diagnóstico sobre la capacidad institucional, organizacional y de gestión de los programas orientada hacia resultados. La finalidad de esta evaluación es proveer información que retroalimente el diseño, la gestión y los resultados de los programas; las de Impacto buscan medir los impactos atribuibles a la operación de los programas. Obtenido de http://www.coneval.gob.mx.

14 Por ejemplo, en materia social, el Coneval registra 5010 programas en su inventario de programas y acciones de desarrollo social: 278 del gobierno federal, 2849 de los gobiernos estatales y 1883 de los municipales. Consejo Nacional de Evaluación de la Política Social, Inventario Nacional de Programas y Acciones de Desarrollo Social, México, 2014.

15 Ibid. 
El mayor beneficio del PBR es la definición de las MIR para los aproximadamente 267 programas federales con reglas de operación, y los 30 del presupuesto rural. Con la MIR, la posibilidad de evaluar resultados en base a objetivos e indicadores definidos, fue un paso fundamental.

El PBR como técnica presupuestaria, termina su vigencia en el transcurso del 2015, y da paso al Presupuesto Base Cero (PBC) a partir del ejercicio fiscal 2016.

\section{6: Presupuesto Base Cero (PBC) y gasto público rural}

\section{¿Qué es el PBC?}

Existe la necesidad de que en la etapa de preparación del presupuesto, opere un mecanismo para examinar cada programa, ubicando objetivos, logros, y justificación de los incrementos en cada ejercicio fiscal: si responden a la inclusión de nuevos componentes dentro de éstos, a la ampliación de la cobertura, a la puesta de marcha de proyectos requirentes de grandes inversiones, o a la introducción de nuevas políticas de desarrollo que conlleven nuevos programas. 16

Los casos de débil asociación entre gasto y resultados de desarrollo han llevado a considerar otros modelos de presupuesto que promueven la evaluación antes de la ejecución y no sólo después de ella.

EI PBC es un esquema que incorpora la evaluación en la fase de preparación del presupuesto y no sólo en la de resultados. En el PBC cada uno de los programas es sometido a una meticulosa revisión que determina si continúa financiándose, si se rediseña, si se incrementa la cantidad de gasto o si se elimina del presupuesto. Esta pre evaluación, toma como criterio la pertinencia del programa con fundamento en las prioridades públicas, la bolsa de recursos con que se cuenta, pero sobre todo, los resultados del programa.

El PBC no toma en consideración lo gastado en ejercicios anteriores o de años pasados para fijar incrementos automáticos; se parte de una estructura en blanco o cero en la que las cantidades de gasto por programa se justifican detalladamente, (lo que, técnicamente, hace del PBC un método muy complicado). El PBC condiciona nuevos incrementos con análisis de programas por ejecutor del gasto.

Las ventajas del PBC en relación a la técnica inercial o incrementalista (en la que subsisten los mismos programas en cada ejercicio fiscal) son que cada una de las actividades está justificada y su financiamiento no depende de los niveles de gasto de presupuestos anteriores; se parte continuamente del por qué se tienen que hacer

16 Las etapas del presupuesto son:

1. Formulación (programación, presentación) 2. Revisión y aprobación 3. Ejecución 4. Control y auditoría 5. Evaluación.

En el caso de México se han agregado las fases de seguimiento antes de la etapa de evaluación, y de rendición de cuentas al final de todo el proceso. Enrique Arriaga, Finanzas Públicas de México (Segunda ed.), México, Instituto Politécnico Nacional, 2001. 
Las reformas que Ilevan al PBC exigen la participación de los tres niveles de gobierno y de la sociedad civil determinados gastos y si la forma en que se están realizando es la más adecuada (alternativas). Uno de los pilares para asegurar la eficiencia es la construcción de paquetes de decisión documentos en los que se presentan las distintas opciones de financiamiento de los programas (lo que también ayuda a localizar intenciones fraudulentas por parte de la burocracia). 17

Las reformas que llevan al PBC no sólo necesitan, sino que exigen la participación de los tres niveles de gobierno y de la sociedad civil en el análisis de la nueva estructura presupuestaria, con mayor razón, en los casos donde las ineficiencias tienen una larga historia. El PBC necesita de buenos diagnósticos previo a su implementación. ${ }^{18}$

\section{Contexto del PBC en México}

El PBC identifica alternativas eficientes de empleo de los recursos lo que responde bien al principio de estabilidad macroeconómica seguido en México. Algunas interpretaciones del PBC resaltan que es un mecanismo que, de no aplicarse fielmente, puede dar paso a adecuaciones indiscriminadas al presupuesto, sobre todo en sistemas donde no hay controles para vigilar a los ejecutores del presupuesto. ${ }^{19}$

El problema de una incorrecta aplicación del PBC, sea por desconocimiento de la metodología, la dificultad para seguirla rigurosamente, o, por la premura, es que puede convertir a esta técnica en una fórmula de adecuaciones presupuestarias laxas en el momento del diseño y la operación de los programas, sin el análisis de raíz que realmente exige. 20

Así ha sucedido con la versión del PBC en México. Mucho antes de que se anunciara la puesta en práctica de este método a inicios del 2015, la Secretaría de Hacienda y Crédito Público (SHCP) venía preparando un recorte al gasto por 124 mil millones de pesos. Es decir, el PBC fue un paso forzado para justificar un nuevo recorte al gasto para el ejercicio fiscal 2016 por 135 mil millones de pesos, más que la consecuencia de buscar una mejor eficiencia del presupuesto. 21

17 Los paquetes de decisión son documentos justificativos que incluyen la información necesaria para que los ejecutores del presupuesto puedan emitir juicios valorativos sobre los programas y su posible integración al presupuesto.

18 Las notas de Carlos Tello, sugieren respecto al tema del "diagnostico" que no es conveniente asumir que el problema de las finanzas públicas y del presupuesto esta sobredianosticado. Pueden existir muchos diagnósticos, pero no buenos diagnósticos. El PBC, como método presupuestario, requiere de la construcción de un diagnóstico de fondo, con la participación de todos los actores. Tello Macías Carlos, Notas sobre Presupuesto Base Cero, México, Cuadernos de Investigación en Desarrollo, UNAM, 2015, pp. 19-25.

19 Oscar Nava Escudero, lo expone con claridad: El PBC puede llegar a ser el medio para que el ejecutivo no le pida permiso a nadie. Foro: Sistema Nacional de Presupuesto. Hacia una reingeniería del ingreso y el gasto público, 12 de agosto del 2015.

20 Las adecuaciones presupuestarias se refieren a todas las modificaciones que se hacen durante el ejercicio fiscal a la estructura financiera y programática de los presupuestos aprobados.

21 Citado en Carlos Tello Macías, Notas sobre Presupuesto Base Cero, op. cit., pp. 14-15. 
Frente a un escenario de caída de los precios del petróleo en el transcurso del 2015 y, en años posteriores, la instauración del PBC en México nace con el sesgo de reducir el gasto. La finalidad del PBC a partir del 2016 es adecuar el recorte del gasto al problema de la baja en los ingresos petroleros puesto que dos terceras partes del financiamiento público provienen de la venta de petróleo. El precio promedio del barril ha seguido una tendencia a la baja desde octubre del 2014 pasando de 100 dólares a 84 para ese mes, y llegando a los 59 en diciembre del mismo año. Para agosto del 2015, el precio llegó a 40 dólares por barril.22

Esa es la racionalidad del ajuste preventivo asociado a la propuesta de poner en práctica el $\mathrm{PBC}$, según lo definido por la Secretaría de Hacienda y Crédito Público (SHCP). Con el PBC se justifica el recorte del gasto y se restringe de manera anticipada, la posibilidad de modificar impuestos, elevar deuda pública y de contrarrestar el carácter pro cíclico de la política fiscal de los últimos años. 23

No es de sorprenderse, ya que la técnica del PBC es trabajosa y complicada si se sigue rigurosamente. En primer lugar, en la etapa de inclusión de programas no hay información completa para el conjunto de programas federales: no todos cuentan con reglas de operación y con MIR para estimar sus resultados; por lo tanto, no hay evaluaciones para todos y se carece de información fiable para tomar decisiones sobre su continuidad o eliminación. En el caso de programas importantes por su naturaleza y gasto, hay un extenso trabajo por parte del Consejo Nacional para la Evaluación de la Política Social (Coneval), la Auditoria Superior de la Federación (ASF), organismos evaluadores independientes y la academia (Por ejemplo, el actual Prospera, antes Oportunidades), pero no es así para la mayoría de programas.

En segundo lugar, la conformación de los paquetes de decisión por La aplicación del PBC ejecutor del gasto en todos los eslabones (secretaría, dependencia, denace primordialmente partamento, enlace), produce grandes cantidades de papeleo y costos de una exigencia derivados de los procesos de análisis, evaluación y priorización de prointerna del gramas; se consume mucho esfuerzo y tiempo, lo que, es una limitagobierno para dar ción respecto a otros métodos cuya relativa sencillez permite sacar los cumplimiento a los proyectos de presupuesto de forma anual sin grandes problemas. Para fines sociales del llegar a esta fase del PBC, se presuponen consensos entre gobierno y Estado sociedad.

Para resumir, el PBC no significa: recorte del gasto, decisión gubernamental de eliminar programas, $y$, reacomodo de programas en corto tiempo y por presiones eventuales. La aplicación del PBC nace primordialmente de una exigencia interna del gobierno para dar cumplimiento a los fines sociales del Estado, a través de un examen sistemático de los programas públicos, pero no se usa para justificar recortes al gasto como consecuencia de ciertos eventos (la baja de los ingresos petroleros, en el caso de México).

El PBC significa: la necesidad de revisar de raíz y a detalle cada programa del presupuesto con fines de mayor calidad y transparencia, ello, con el propósito ge-

22 Obtenido de: www.economia.gob.mx, 25 de agosto del 2015.

23 Carlos Tello Macías, Notas sobre Presupuesto Base Cero, op. cit. 


Gráfica 1
Montos del Programa Es-
pecial Concurrente (PEC) y
su participación porcentual
en el gasto programable
del Sector Público Presu-
puestario (SPP)

neral de recuperar el papel del gasto en el desarrollo a través de la inversión en renglones de impulso al crecimiento y redistribución del ingreso.

\section{Situación actual del gasto público rural}

A partir de la introducción de la nueva Ley de Desarrollo Rural Sustentable (LDRS) en 2001, y la creación del Programa Especial Concurrente para el Desarrollo Rural Sustentable (PEC), los montos de gasto público se multiplicaron por cuatro (pasando de $\$ 71000$ millones de pesos constantes a $\$ 310$ 000) entre 2001 y 2013 (gráfica 1).

Tanto la norma como el instrumento, se tradujeron en una asignación de gasto mayor. En el PEC se concentraron todas las secretarías y programas de naturaleza social y productiva dirigidos al campo, cuyo gasto correspondía previamente a la Secretaría de Agricultura, Ganadería, Desarrollo Rural, Pesca y Alimentación (Sagarpa).

También, siguiendo los principios de coordinación y concurrencia, el PEC organizó en diez vertientes sus más de cien programas en operación: 1) Agraria; 2) Financiera; 3) Laboral; 4) Administrativa; 5) Medio ambiente; 6) Salud; 7) Educativa; 8) Social; 9) Infraestructura; 10) Económica.

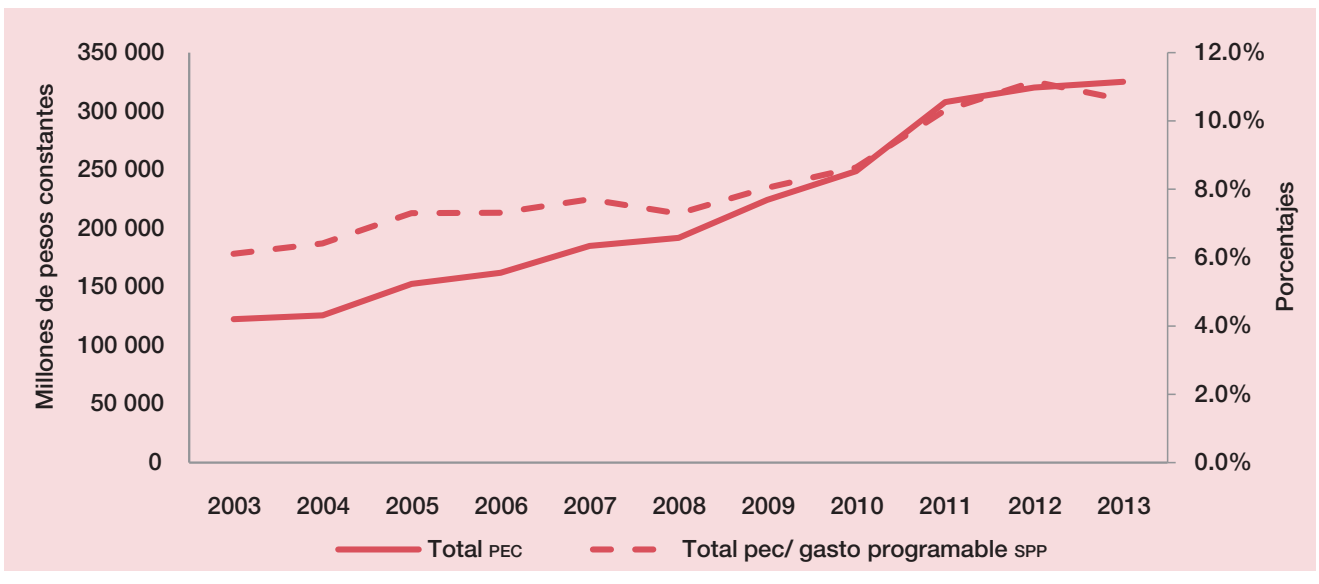

Fuente: elaborado con base en Centro de Estudios para el Desarrollo Rural Sustentable y la Soberanía Alimentaria (CEDRSSA) de la Cámara de Diputados; Proyecto de Presupuesto de Egresos de la Federación, varios años.

Actualmente, la principal crítica al PEC, proveniente tanto de las condiciones generales de las zonas rurales, como de la conclusión de las evaluaciones disponibles, es que no representó más que un nuevo modelo contable para la organización de los distintos programas, útil en la comprensión del esquema presupuestario, pero desprovisto de una estrategia de desarrollo.

La integración de 17 secretarías en el ejercicio del gasto, no mejoró la coordinación, ni la sinergia de programas, pues la mayoría opera de forma aislada e incluso contradictoria a los objetivos de igualdad y crecimiento. ${ }^{24}$ Recursos cuya inversión

24 Como ejemplo, se puede citar el caso del Programa Procampo (ahora Proagro Productivo), el cual, debido a su diseño de entrega del apoyo monetario por superficie de tierra, benefició a 
puede resultar en mejoras significativas incurren en un alto costo de oportunidad como efecto de la dispersión de funciones y el sostenimiento de programas regresivos o poco productivos.

Así lo muestran los indicadores de desarrollo. En la distribución del ingreso total rural (ITR) por deciles, a los tres primeros les corresponde un promedio de $3.1 \%$ del ITR, mientras que a los deciles 9 y 10 , un promedio de $25.5 \%$ del ITR; respecto a la pobreza, $66.8 \%$ de la población rural total está en pobreza (16.7 millones de personas de un total de 25 millones); y, en cuanto al PIB agropecuario, su tasa de crecimiento de $1.9 \%$ en los últimos treinta años, es baja (crisis crónica).

El punto más importante del PBC es la característica democrática En el medio rural el de su puesta en marcha, difícil de cumplir en el escenario de inercias ciclo de reproducción y presiones existente en México, pero particularmente importante, en de la pobreza continúa el medio rural donde a pesar de la recomposición del gasto a favor de y no se aprecia el programas sociales, el ciclo de reproducción de la pobreza continúa y trabajo de la CIDRS no se aprecia el trabajo de la Comisión Intersecretarial para el Desarrollo Rural Sustentable (CIDRS),25 ni de los 127 organismos sociales, privados, productivos y de investigación, que conforman el Consejo Mexicano para el Desarrollo Rural Sustentable (CMDRS).

La implicación para el gasto rural de la versión PBC de recorte al gasto, será la resectorización o fusión de los programas en operación. No se logrará la revisión programa por programa y la inclusión de la opinión social, como lo marca la metodología del PBC.

Es difícil que se terminé con la forma de hacer política rural en México en la que no hay una estrategia, y predomina la práctica difusa del conjunto de programas, la

productores grandes y no a los pequeños. Fue uno de los programas más regresivos del presupuesto rural.

25 En el artículo 21 de la Ley de Desarrollo Rural Sustentable de 2001 quedaron registradas las 10 secretarías encargadas del presupuesto rural:

1. Secretaría de Agricultura, Ganadería, Desarrollo Rural, Pesca y Alimentación (Sagarpa) cuyo titular preside la CIDRS;

2. Secretaría de Economía (SE);

3. Secretaría de Medio Ambiente y Recursos Naturales (Semarnat);

4. Secretaría de Hacienda y Crédito Público (SHCP);

5. Secretaría de Comunicaciones y Transportes (SCT);

6. Secretaría de Salud (ss);

7. Secretaría de Desarrollo Social (Sedesol);

8. Secretaría de la Reforma Agraria (SRA);

9. Secretaría de Educación Pública (SEP);

10. Secretaría de Energía (Sener).

Para el 2007, el número de secretarías aumentó a 17, incorporándose: la Secretaría de Gobernación (Segob); el Instituto Nacional de las Mujeres (Inmujeres); el Instituto Nacional para el Federalismo y el Desarrollo Municipal (Inafed); Financiera Rural (Finrural); la Comisión Nacional del Agua (Conagua); la Conferencia Nacional de Gobernadores (Conago); y la Asociación Mexicana de Secretarios de Desarrollo Agropecuario, A.C. (AMSDA). Cámara de Diputados, Ley de Desarrollo Rural Sustentable, México, 2001. Véase también: Angélica Tacuba Santos, Gasto público y desarrollo rural en México, 1990-2013, México, Tesis de Doctorado del Posgrado de Economía, UNAM, 2015. 
dispersión de funciones entre secretarías, la solicitud de incrementos de gasto sin confrontar por resultados, la inexistencia de prioridades regionales (norte, sur) y el uso clientelar de los programas sociales. Un presupuesto ejercido en esas condiciones, pierde su esencia de instrumento de desarrollo, independientemente de la técnica seguida.

\section{Ejercicio hipotético de aplicación del $P B C$ al presupuesto rural}

Dando por sentado que la versión del PBC en México no se ajustará a lo establecido, pero al mismo tiempo, haciendo un ejercicio que permita visualizar lo que sucedería si se cumpliera con rigurosidad, la aplicación del PBC al gasto rural quedaría de la siguiente manera.

El primer paso sería el consenso sobre la prioridad del desarrollo rural y la definición de acciones que conducirían a la revisión del principal instrumento del gobierno para ese fin, el PEC. Se abordaría con ello su principal contradicción que es el distanciamiento entre los principios de coordinación y concurrencia de programas y la ausencia de los mismos en los años que lleva de operar.

El segundo paso, sería la definición de objetivos sectoriales: productivos, sociales, ambientales; de las secretarias de la CIDRS, y sus programas. En esta etapa se exigiría, la justificación detallada de cada uno de ellos y de todos sus componentes (un solo programa puede tener varios componentes o sub renglones de gasto).

Seguiría la integración de los paquetes de decisión, en los que cada programa es debidamente justificado, pasándose a revisión desde los niveles más bajos hasta los más altos de cada secretaría. En esta misma etapa, los niveles superiores iniciarían un trabajo de retroalimentación hacia abajo, en el que, se expondrían observaciones y sugerencias a los paquetes presentados.

Finalmente, una vez salvados los paquetes de decisión, y, suponiendo una base de información sobre el desempeño de cada programa y componente de gasto, se determinaría qué programas incluir en el presupuesto. El escenario más probable es que el número de programas existentes disminuyera - aunque no drásticamentecomo efecto del escrutinio a fondo de los resultados, particularmente, en los casos de regresividad, los que duplican acciones y los que se integran sin justificación (en especial, subsidios agrícolas) (gráfica 2). 


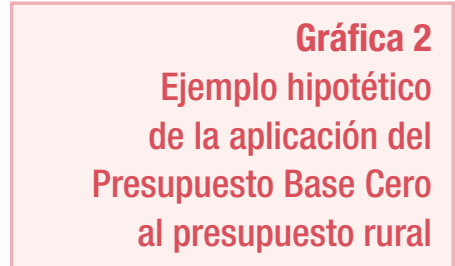

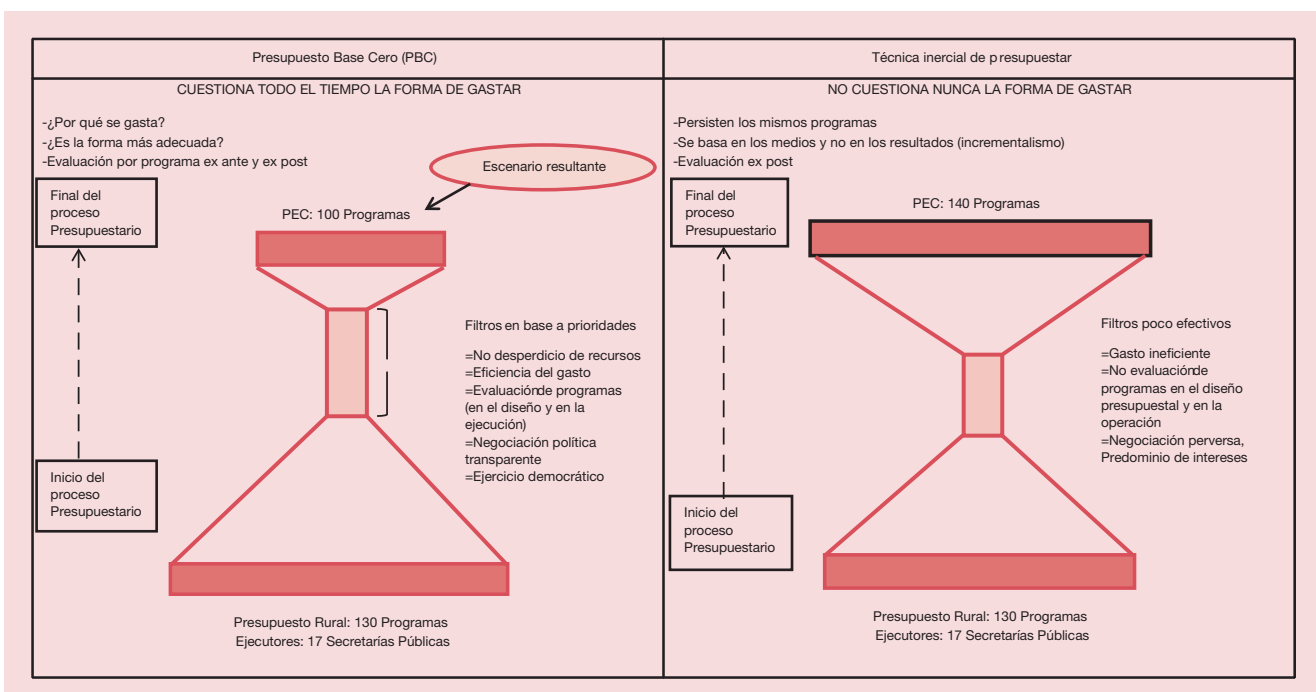

Fuente: elaborado con base en los fundamentos del Presupuesto Base Cero (PBC).

\section{Por pasos, el camino sería:}

1. Diagnóstico a fondo de la situación general del gasto rural y de cada uno de sus programas, con la finalidad de generar información para la inclusión-exclusión, o rediseño;

2. Involucramiento de los sectores sociales (organizaciones, sociedad civil) en el debate y nueva estructura programática del presupuesto;

3. Acogimiento del PBC como nuevo esquema presupuestario por cada dependencia y conocimiento de la metodología;

4. Coordinación al interior de las 17 secretarías de la CIDRS para revisar el inventario de programas, objetivos y logros (en especial la Sagarpa, la SEP y la Sedesol, que ejercen $58.6 \%$ del total del presupuesto rural);

5. Conformación de los paquetes de decisión integrados por: la lista de programas, confrontación de objetivos y resultados, alternativas en el diseño y ejecución de cada uno, y la debida justificación para su permanencia;

6. Traslado de los paquetes de decisión a los niveles superiores de cada secretaría quienes deben revisar y hacer las observaciones necesarias, y devolverlos a los niveles inferiores;

7. Atención de los niveles inferiores a las observaciones realizadas por los superiores (este proceso se repetirá hasta que se tenga un paquete de decisión lo suficientemente coherente para presentarlo a la federación);

8. Conformación de un solo paquete de decisión por secretaria (en el caso rural serían 17 paquetes en total);

9. Presentación del paquete de decisión a la federación para su análisis e inclusión en el presupuesto nacional; 
10. Revisión detallada por parte de la federación de los paquetes de decisión de cada secretaría y aceptación o rechazo de las propuestas (para aproximadamente 130 programas del presupuesto rural).

Como puede observarse, el tiempo requerido para cada uno de los puntos agota por completo la anualidad del presupuesto y exige un esfuerzo gigantesco por parte de cada secretaría en tiempo, coordinación interna, recursos financieros, materiales y la participación de evaluadores externos para vigilar el proceso. Un recorrido así, no será posible en el transcurso del 2015, año en que se anuncia la aplicación del PBC y se inicia la revisión de los programas federales casi a mediados de año; 26 no será posible para 17 dependencias públicas ejecutoras del gasto rural y para más de 100 programas rurales con diversos componentes y sub renglones.

Además, el seguimiento riguroso de la metodología PBC traería adjunta la depuración del proceso presupuestario en lo administrativo y técnico, pero también en lo político. Acarrearía un nuevo escenario de negociación entre gobierno, partidos y beneficiarios de los programas; así como, la disminución del uso clientelar y rentista del presupuesto.

Ello, supondría una dificultad extraordinaria en un medio rural cercado de fortalezas e instituciones informales que aprovechando la pobreza, han asentado un agresivo uso clientelar de los programas sociales, con la cooperación del mismo gobierno, los partidos y algunos sectores rurales. No debemos omitir que en la administración del presupuesto rural, la burocracia actúa bajo intereses, esquemas racionales de maximización de beneficios y controlando recursos públicos en condiciones de monopolio.

Sin embargo, aunque la aplicación del PBC no es sencilla, la propuesta que hace de revisar cómo se gasta sería lo suficientemente aceptable al concentrarse en la trayectoria del desarrollo rural, que muestra un vacío entre cantidades de inversión pública y buenos resultados.

\section{Conclusion}

La puesta en práctica de la versión original del PBC para el próximo 2016 no da señales de factibilidad política y técnica. A pesar de vislumbrarse como un gran paso, no deja claro que su aplicación sea posible por todo lo que técnicamente supone el PBC para un sinnúmero de programas federales.

Pero además, porque el severo método supondría cancelar de tajo la cultura clientelar del gasto que domina y los grupos de interés que se benefician de ella, lo

26 Al respecto, el Centro de Estudios de las Finanzas Públicas de la Cámara de Diputados (CESFP) convocó para el día 25 de marzo de 2015 a un foro con la siguiente leyenda: "La H. Cámara de Diputados a través del Centro de Estudio de las Finanzas Públicas, conjuntamente con el Banco Mundial y la Unidad de Posgrado de Economía de la UNAM, llevará a cabo el 'Foro Internacional sobre el Presupuesto Base Cero', en razón de que el Ejecutivo Federal planteó que la elaboración del Proyecto de Presupuesto de Egresos 2016 se llevará a cabo bajo la metodología de esta técnica presupuestal". Obtenido de: http://www.cefp.gob.mx. 
que no es del todo conveniente para la racionalidad partidista del gobierno que subyace al presupuesto.

En términos de la Escuela de la Elección Pública (EEP), la aplicación estricta del PBC equivaldría a tener un Estado benevolente, con fines colectivos predeterminados e inviolables, y sin rent seekers, un rasgo, difícil de construir bajo el sentido de política que se esconde tras el presupuesto, aunque, tampoco es inalcanzable con un marco institucional íntegro que aplaque el oportunismo y la corrupción. 27

La teoría de las instituciones de North da luz sobre los cambios normativos y su éxito: aunque las normas formales puedan cambiar de la noche a la mañana como resultado de decisiones políticas o judiciales, las limitaciones informales (costumbres, conductas, tradiciones) son una barrera. El cambio institucional genuino involucra incursiones decisivas de las leyes en tales fortalezas para erradicar modelos de conductas erróneos que son obstáculos para cualquier reforma. 28

El seguimiento de la versión PBC de disminución del gasto, seguramente entrañará, a escala rural, una revisión de los programas no tan rigurosa como debiera, pero pondrá sobre la mesa de análisis dos de los puntos más importantes del desarrollo rural y la política pública:

1) No hay concurrencia entre secretarías ejecutoras del gasto. Las 17 secretarias de la CIDRS enfrentan como principal problema, la ausencia de una estrategia nacional de desarrollo rural, que resulta en una política pública difusa y de bajo impacto.

2) No hay sinergia entre programas. Los aproximadamente 130 programas del presupuesto rural operan desarticulados duplicando funciones o sin definir claramente objetivos, población, y problemática por atender.

Estos dos aspectos son las fragilidades a resolver si además de las ventajas del PBC como método presupuestario, se tiene como objetivo primordial el desarrollo de las zonas rurales donde habitan 25 millones de personas, la mayoría de ellas, en pobreza. Hasta ahora, el desarrollo humano como proceso de mejoramiento de las capacidades integrales de la población, está atascado en una política pública discordante y de gasto creciente sin un análisis en el diseño de programas y de sus resultados, lo que reduce la eficacia instrumental del gasto público en la promoción del crecimiento y la redistribución del ingreso.

27 La escuela de la Elección Pública en una crítica a la teoría de los fallos del Estado comparte con el neoinstitucionalismo la premisa del individualismo. Su tesis es que burócratas y políticos no priorizan el bienestar social y son maximizadores de su interés mediante el presupuesto público.

28 Douglass North, Instituciones, cambio institucional y desempeño económico, México, Fondo de Cultura Económica, 1993. 


\section{Bibliografía}

Arellano, David (2000), Presupuesto dirigido a resultados: los dilemas de la evaluaciòn por desempeño en contextos latinoamericanos. Leccio-

nes desde México, Santo Domingo: Congreso Internacional del CLAD.

Arriaga, Enrique (2001), Finanzas Públicas de México (Segunda ed.), México: Instituto Politécnico Nacional.

Barea, José, \& Martínez, José. Antonio (2004), Fortalezas y debilidades del Presupuesto Base Cero, Instituto Latinoamericano y del Caribe de Planificación Económica y Social.

Hirschman, Albert (1989), Enfoques alternativos sobre la sociedad de mercado y otros enfoques recientes,

México: Fondo de Cultura Económica.

Keynes, John (2000), Teoría general de la ocupación, el interés y el dinero, México: Fondo de Cultura Económica.

Meny, I., \& Thoenig, J. (1992), Las políticas públicas, Madrid: Ariel.

Musgrave, Richard (1973), Principios de determinación del presupuesto, En, Francisco Domínguez, Política fiscal en acción, Madrid: Instituto de Estudios Fiscales.

North, Douglass (1993), Instituciones, cambio institucional y desempeño económico, México: Fondo de

Cultura Económica.

Novelo, Federico (1997), Invitación a Keynes, México: Universidad Autonóma Metropolitana, Fondo de Cultura Económica.

Serrano, José Antonio (2001), La naturaleza de las politicas públicas, Santiago: Universidad Pontificia de Chile.

Shack, Nelson (2008), Intentanto caracterizar la articulación entre Plan y Presupuesto. Algunas experiencias en América Latina, Banco Interamericano de Desarrollo (BID).

Smith, Adam (2002), La riqueza de las naciones, Madrid: Alianza Editorial.

Tacuba, Angélica (2015), Gasto público y desarrollo rural en México, 1990-2013, México: Posgrado de Economía UNAM.

Tello, Carlos (2015), Notas sobre presupuesto base cero, Cuadernos de Investigación en Desarrollo, México. 